\title{
Puck satellite Earth-based observations ${ }^{\star} \star \star$
}

\author{
C. H. Veiga ${ }^{1}$ and P. Bourget ${ }^{2}$ \\ 1 Observatório Nacional, Rua General José Cristino 77, 20921-400 Rio de Janeiro, Brazil \\ e-mail: cave@on.br \\ 2 Service d'Aéronomie du CNRS, BP 3, 91371 Verrières-le-Buisson Cedex, France
}

Received 7 February 2006 / Accepted 24 March 2006

\begin{abstract}
Puck, a faint satellite very close to Uranus' planet, was discovered by Voyager 2 Spacecraft images in 1986. Ever since then, few observations from Earth have been made. This prompted us to start a program of systematic observations of this satellite with the $1.6 \mathrm{~m}$ telescope at the Laboratório Nacional de Astrofísica/MCT (Itajubá, Brazil). The success of the observations is mainly due to the use of a Coronagraph developed at Observatório Nacional/MCT (Rio de Janeiro, Brazil). This article presents astrometric positions obtained from Earth observations of Puck and of the five major Uranian satellites for four nights in 2004. Those positions are compared to the theoretically calculated positions from JPL Development Ephemeris. For Puck, the root mean square (rms) of the mean residual was found to be 84 milliarcseconds (mas). The stars' USNO-A2.0 catalog was used as a reference system for the astrometric calibration.
\end{abstract}

Key words. planets and satellites: general - celestial mechanics - astrometry

\section{Introduction}

Puck is one of the ten small faint inner moons of Uranus discovered by Voyager 2 Spacecraft in 1986 (Smith et al. 1986). Its revolution period is 0.769 days, its diameter $154 \mathrm{~km}$, and its mass unknown. The maximum elongation at mean opposition is 6!"3, corresponding to an average distance of $86004 \mathrm{~km}$ from Uranus. Puck is very dark due to its low albedo (0.1) (Owen et al. 1987).

For us, the extreme differential visual magnitude at opposition between Uranus $(V=5.5 \mathrm{mag})$ and Puck $(V=20.2 \mathrm{mag})$ and their closeness, along with to the atmosphere from Earth, are the main problems for Earth-based observation. Thus, faint satellites close to bright planets have their images embedded in the planet's halo. This fact doesn't allow observation of the satellite or else it doesn't allow precise measurent of the image's center.

In our case, the greatest difficulty for these observations was that we did used neither a large ground-based telescope nor adaptive optics system to correct the turbulence effect from Earth atmosphere. The development of a new Coronagraph for this difficult planetary observation was the solution for obtaining precise positions of Puck.

During winter of the southern hemisphere, when local metereological conditions are more favorable in Brazil, 135 CCD observations of the Puck satellite were carried out for four nights in 2004. Measurable images of Ariel, Umbriel, Titania, Oberon, and Miranda were also obtained on the same CCD frames.

\footnotetext{
* Based on observations made at the Laboratório Nacional de Astrofísica/MCT-Itajubá-Brazil.

$\star \star$ The catalog is only available in electronic form at the CDS via anonymous ftp to cdsarc.u-strasbg.fr (130.79.128.5) or via http://cdsweb.u-strasbg.fr/cgi-bin/qcat?J/A+A/454/683
}

\section{The observations}

One hundred thirty-five Puck CCD Earth-based observations were made at the Cassegrain focus of the $1.6 \mathrm{~m}$ RitcheyChretien reflector (Perkin-Elmer) of the Laboratório Nacional de Astrofísica (LNA/MCT) Itajubá-Brazil, with geographical coordinates of longitude: $3^{\mathrm{h}} 02^{\mathrm{m}} 19^{\mathrm{s}}$, latitude: $-22^{\circ} 32^{\prime} 04^{\prime \prime}$, and altitude: $1872 \mathrm{~m}$ (IAU reference number 874 ). The focal distance of the Cassegrain combination is equal to $15.8 \mathrm{~m}$, and the focal plane is 13 .' $^{\prime} / \mathrm{mm}$ (see Veiga et al. 1995).

To take the 135 Puck images, we used the SITe SI003AB CCD type, back-illuminated, with an array of $1024 \times 1024$ square pixels corresponding to $24 \mu \mathrm{m}, 0^{\prime \prime} 312$ on the sky. No filter was used and the exposure time varied from 20 to $120 \mathrm{~s}$, depending on the meteorological conditions. The sky quality (seeing) varied between $0{ }^{\prime} 8$ and $1^{\prime \prime} 2$ during the four nights of observation.

Due to its faintness and its proximity to scattered light from the planet, Puck is very difficult to be observed. In Pascu et al. (1998), thirty-three images were obtained with Hubble Space Telescope (HST) observations. Ariel and Miranda's positions were used to determine the scale and orientation of the CCD. Puck positions are relative to Miranda. Jacobson (1998) uses eighty-one observations (32 HST and 49 Voyager 2 Spacecraft data) to revise the complete set of orbital elements. Recent Earthbased observations, published in Descamps et al. (2002), by using an Adaptive Optics system mounted on the ESO-La Silla $3.6 \mathrm{~m}$ telescope, did not show the number of observed positions clearly.

To observe this satellite with a classical optics telescope of $1.6 \mathrm{~m}$ and still without an adaptive optics system would be an impossible mission. To overcome this difficulty, we used a diaphragm with eight circular apertures between the secondary support vanes (Veillet et al. 1980). The diffraction spikes on Uranus's image can occult the image of Puck. The decrease of nearly $40 \%$ in the useful area of the primary mirror was 
compensated by an increase in the exposure time. However, that does not avoid the diffusion of light around the planet, caused mainly by Earth atmospheric turbulence. The big differential magnitude between them is another serious problem.

Then, to both solve that and improve the accuracy of the satellite position, we used a Coronagraph especially designed with the astrometric purpose of imaging faint satellites near planets. In order to optimize the classical process idealized by Lyot (1939), a Lyot mask with a variable-diameter occulting disk (Hg mask) was developed. This process facilitates a direct detection of Puck image in each CCD frame. The addition of several frames is not necessary to capture a faint image of the satellite. For more details see Bourget et al. (2001).

\section{Astrometry}

In order to find the center of satellites and star images on the CCD, each position was determined by a two-dimensional Gaussian fitting a circular area around the images. The algorithm is based on the adjustment of a point-spread function, and a second-degree polynomial is also adjusted in order to remove the background contaminated by the light from the planet. This background removal is essential to avoid systematic errors in the measurements of the satellites centers.

For Puck images, a small circular area was used due to the very small number of pixels and its proximity to the image of the $\mathrm{Hg}$ mask on the CCD frame. The error in the image's determination center of Puck is 7 mas; for the five other satellites it is 4 mas, and for the stars field 2 mas. The measuring process was automated using the language INTERPOL, inside of the ASTROL routine package (Colas \& Serrau 1993).

Due to the poor stellar field in our observations (about 14 stars on CCD) and magnitudes between 18 and 20, it was not possible to use the best choice, the high-accuracy UCAC2 star catalog in the astrometric calibration. In spite of UCAC2 having 48 million stars covering the sky from -90 to +52 degrees in inclination (Zacharias et al. 2004), its magnitude is limited to between 7.5 and 16. Therefore, we were forced to use the classical process of astrometric calibration to determine the observed coordinates of the satellites in the sky. The number of stars in each CCD field was enough to allow us to carry out a classical astrometric reduction.

In this process the USNO-A2.0 stars catalog (Monet et al. 1998) was used as a reference system, and the UCAC2 catalog was used to correct the local systematic errors of the USNO-A2.0. The stars' positions are referred to the ICRS (International Celestial Reference System) and given at the epoch J2000.0. A least-square procedure was used with those positions based on the Householder transformation (Lawson \& Hanson 1974) to fit the transformation parameters. Using the corrected coordinates of the USNO-A2.0 stars in the frame, we determined the frame constants with a first-degree polynomial (six-constant model). This process is iterative and at every step, we eliminated the stars with residuals larger than 1000 mas. The standard deviation characteristic of the residuals of the remaining stars is about 120 mas. With the frame parameters defined (scale and orientation), we computed the equatorial coordinates of the Uranian satellites.

In the list, available only in electronic form, the topocentric observed positions of Ariel, Umbriel, Titania, Oberon, Miranda, and Puck are referred to a mean equator and equinox J2000 system. The time is given in year, month, and decimals of the day in Universal Time. The coordinates' right ascension and
Table 1. Extract of the list of observations available only in electronic form. Starting from Col. 1 we have: the international code adopted for Uranus satellites; the time in year, month, and decimals of the day in Universal Time; and the coordinates' right ascension and declination, given in degree and fraction. These topocentric observed positions of six satellites refer to a mean equator and equinox J2000 system.

\begin{tabular}{|c|c|c|c|}
\hline Code & Time & Right ascension & Declination \\
\hline 701 & 2004820.1558403 & 337.1817259 & -10.4040744 \\
\hline 702 & 2004820.1558403 & 337.1812407 & -10.4031928 \\
\hline 703 & 2004820.1558403 & 337.1802004 & -10.3983271 \\
\hline 704 & 2004820.1558403 & 337.1865410 & -10.4159758 \\
\hline 705 & 2004820.1558403 & 337.1828225 & -10.4035270 \\
\hline 715 & 2004820.1558403 & 337.1826277 & -10.4048603 \\
\hline
\end{tabular}

Table 2. Observed minus calculated statistics observations of Puck satellite for each night. $N_{\text {obs }}$ is the final number of observations. $\bar{N}_{\text {stars }}$ is the mean number of reference stars used in the astrometric calibration. The quantities $\bar{\alpha}, \sigma_{\bar{\alpha}}, \bar{\delta}, \sigma_{\bar{\delta}}$ are the means and the standard deviations for the residuals in right ascension and declination. The two last columns are the rms of the residuals in the right ascension and declination directions. The units are in milliarcseconds.

\begin{tabular}{rcccccccc}
\hline \hline 2004/Aug./Day & $N_{\text {obs }}$ & $\bar{N}_{\text {stars }}$ & $\bar{\alpha}$ & $\sigma_{\bar{\alpha}}$ & $\bar{\delta}$ & $\sigma_{\bar{\delta}}$ & $\mathrm{rms}_{\bar{\alpha}}$ & $\mathrm{rms}_{\bar{\delta}}$ \\
\hline 20 & 26 & 17 & 28 & 130 & 9 & 87 & 133 & 88 \\
21 & 67 & 15 & 8 & 70 & -51 & 75 & 71 & 91 \\
22 & 26 & 13 & -4 & 58 & 12 & 60 & 59 & 62 \\
23 & 16 & 13 & -16 & 69 & -10 & 77 & 71 & 79 \\
\hline Total & 135 & & 6 & 84 & -22 & 81 & 84 & 84 \\
\hline
\end{tabular}

declination are given in degree and fraction, with seven significant digits.

Based on the international code, adapted to Uranus's satellites, starting from Col. 1 of the list, we have: the number of the satellite, the date of the observation, topocentric observed right ascension, and declination. Table 1 here provides a brief extract of the electronic list.

\section{Comparison with theoretical positions}

In this section the observed positions of the six satellites are compared to their theoretical positions. The ephemerides used in this work are available electronically from the JPL Horizons on-line solar system data and ephemeris computation service (Giorgini et al. 1996). The analytic model is based on the OS87 theory, namely, a precessing ellipse referred to the Uranian equator (Jacobson 1998).

In order to evaluate the accuracy of our observations for each night, Table 2 shows the main results of this paper, $\Delta \alpha$ and $\Delta \delta$ residual means, and the standard deviation of Puck. From Table 2 we can see the mean of the observed minus-calculated residuals for Puck: $\Delta \bar{\alpha}=6$ mas and $\Delta \bar{\delta}=-22$ mas and the standard deviations, $\sigma_{\bar{\alpha}}=84$ mas and $\sigma_{\bar{\delta}}=81$ mas. The rms of the Puck residuals is about 84 mas in the right ascension and declination directions.

Figure 1 presents the distribution of Puck positions $\Delta \alpha \cos \delta$ and $\Delta \delta$ on the theoretical orbit at the epoch of the observations. Figure 2 displays the residual $\Delta \alpha$ and $\Delta \delta$, in milliarcseconds, as a function of time.

The result from August 20 is not as precise as the ones on the other three nights. It shows the difficulty of observing this satellite very close to the planet (see Fig. 1). On the other hand, this result is relevant because the observed positions in this orbit 


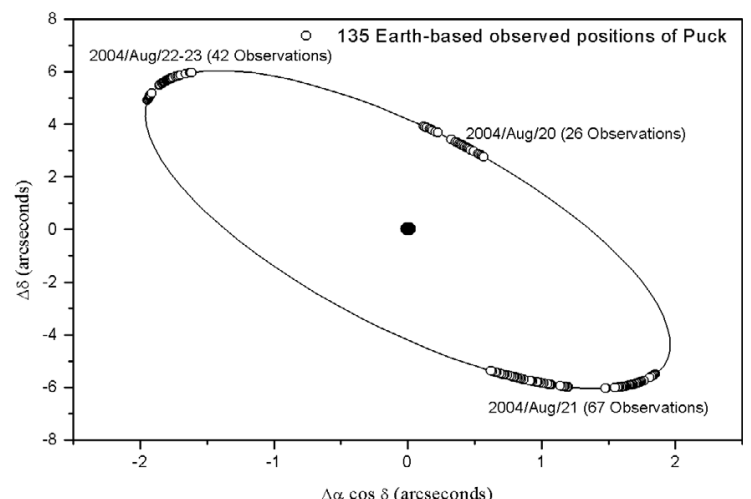

Fig. 1. The observed positions of Puck in its orbit for each night. The positions are represented by circles. The final number of observations is in brackets.
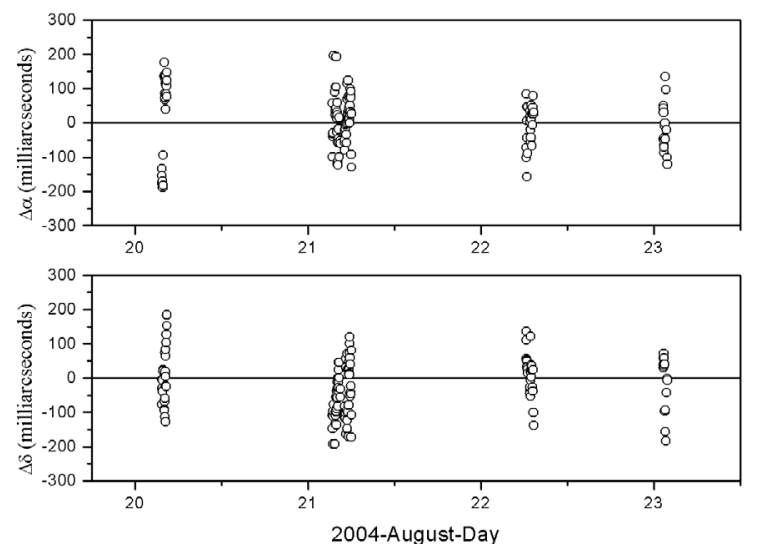

Fig. 2. Residuals, in milliarcseconds, for the Puck satellite as a function of time. The parameters $\Delta \alpha$ and $\Delta \delta$ are $\left(\alpha_{\text {observed }}-\alpha_{\text {calculated }}\right)$ and $\left(\delta_{\text {observed }}-\delta_{\text {calculated }}\right)$, respectively.

region can contribute significantly to a future improvement in our understanding of Puck's orbit.

Also in Fig. 1, the observations between August 21 and 23 were made when Puck had a mean elongation of 5'.9, near maximum elongation. The observations of August 20 were made very close to the planet (elongation $=3$.' 3 ). In this situation it is very difficult to observe this satellite. However, even for this orbit region, we obtained positions with good accuracy.

The comparison between the observed and calculated positions, right ascension, and declination, of Ariel, Umbriel, Titania, Oberon, and Miranda are presented in Table 3. This table gives the final number of observations and the mean and standard deviation in milliarcseconds for all nights.

\section{Concluding remarks}

In this article we presented 135 Earth-based CCD positions of Puck satellite, referring to a mean equator and equinox
Table 3. Observed minus calculated statistics for all observations of Ariel, Umbriel, Titania, Oberon, and Miranda. $N_{\mathrm{obs}}$ indicates the final number of observations. The quantities $\bar{\alpha}, \sigma_{\bar{\alpha}}, \bar{\delta}, \sigma_{\bar{\delta}}$ are the means and the standard deviations for the residuals in right ascension and declination. The units are in milliarcseconds. The mean number of reference stars used here is the same as in Table 2.

\begin{tabular}{cccccc}
\hline \hline Others satellites & $N_{\text {obs }}$ & $\bar{\alpha}$ & $\sigma_{\bar{\alpha}}$ & $\bar{\delta}$ & $\sigma_{\bar{\delta}}$ \\
\hline Ariel (701) & 46 & 8 & 96 & -7 & 33 \\
Umbriel (702) & 82 & -40 & 89 & 6 & 63 \\
Titania (703) & 88 & -46 & 97 & 47 & 65 \\
Oberon (704) & 37 & -40 & 54 & -98 & 36 \\
Miranda (705) & 34 & 40 & 85 & -8 & 27 \\
\hline
\end{tabular}

J2000 system. These observations were made during four nights of 2004 on the classical $1.6 \mathrm{~m}$ Ritchey-Chretien reflector and without an adaptive optics system. The results, shown in the section above, confirm that our CCD observations, the measurements, and the astrometric calibration system allowed us to determine precise positions. The distribution of our residuals in Fig. 2 and the result in Table 2 show good agreement with the Jacobson ephemeris.

These new positions can help better to determine the parameters of Puck's orbit. The success of the observations is mainly due to the efficiency of the Coronagraph with a variable-diameter occulting disk. The main purpose of this work has been to collaborate on our series of astrometric Earth-based observations and to join them to all the others of the Hubble Space Telescope and Voyager 2 Spacecraft to improve accuracy on the ephemerides of Puck.

Acknowledgements. The authors thank all colleagues at the Laboratorio Nacional de Astrofísica/MCT for their efforts and help during the observations. C.H. Veiga wishes to thank the National Council for Scientific and Technological Development $(\mathrm{CNPq})$ of the Brazilian Ministry of Science and Technology (MCT) for partial support for this work, and the State of Rio de Janeiro Science Foundation-FAPERJ for providing computational facilities for this project.

\section{References}

Bourget, P., Veiga, C. H., \& Vieira Martins, R. 2001, ASP, 113, 436 Colas, F., \& Serrau, M. 1993, Astrol and Interpol version 3.10. (Paris, France: Édition du Bureau des Longitudes)

Descamps, P., et al. 2002, C. R. Phys. 3, 121, 128

Giorgini, J. D., et al. 1996, BAAS, 28, No. 25.04

Jacobson, R. A. 1998, AJ, 115, 1195

Lawson, C. L., \& Hanson, R. J. 1974, Solving Least Squares Problems (Englewood Cliffs: Prentice-Hall)

Lyot, B. 1939, MNRAS, 99, 580

Monet, D., Bird., A., et al. 1998, The USNO-A2.0 Catalogue (Washington DC: US Naval Observatory)

Owen, W. M., \& Synnott, S. P. 1987, AJ, 93(5), 1268

Pascu, D., Rohde, J. R., Seidelmann, P. K., et al. 1998, AJ, 115, 1190

Smith, B. A., Soderblon, L. A., Bube, R., et al. 1986, Science, 233, 43

Veiga, C. H., \& Vieira Martins, R. 1995, A\&AS, 113, 557

Veillet, C., \& Ratier, G. 1980, A\&A, 86, 342

Zacharias, N., Urban, S. E., Zacharias, M. I., et al. 2004, AJ, 127, 3043 